\title{
A combined chemo-mechanical approach for aesthetic management of superficial enamel defects
}

\author{
S. Ardu, ${ }^{1}$ N. Benbachir, ${ }_{1}^{2}$ M. Stavridakis, ${ }^{3}$ D. Dietschi, ${ }^{4}$ I. Krejci ${ }^{5}$ \\ and A. Feilzer ${ }^{6}$
}

Objective The aim of this article is to describe an easy technique for managing small superficial defects in light to medium fluorosis. Method and materials The proposed technique is based on a selective abrasion of the superficial enamel and a recreation of the superficial macro and micro morphology. The aesthetic appearance can be enhanced by power or home bleaching. Results The presented technique can manage enamel defects which are confined in the most external enamel surface with satisfying aesthetic results. Conclusions This conservative approach may be considered an interesting alternative to more invasive prosthetic techniques based on composite reconstructions or ceramic veneers, minimising invasivity, chairside time and costs for patients.

\section{INTRODUCTION}

In recent decades, due to fluoridation of drinking water and the addition of fluoride to milk and salt, fluorosis has increased in western countries. ${ }^{1-3}$ This kind of pathology leads to the whitish, opaque, unpleasant appearance of enamel which is often visible at speaking distance. Proposed treatments, depending on fluorosis severity, ${ }^{4}$ range from expensive ceramic veneers to free hand bonding restorations and abrasive chemical treatments.

Although aesthetic demands for pleasing, brighter smiles are steadily increasing, economic problems are also concerning more of the population. Dentists therefore have the dilemma of being obliged to create highly aesthetic results even if a patient's means are rather limited. More conservative approaches based on cheaper and less time-consuming treatments such as bleaching,

$1^{*}-4$ Lecturers, Department of Cariology \&t Endodontology, Dental School, University of Geneva, Geneva, Switzerland; ${ }^{5}$ Professor and Chairman, Department of Cariology \& Endodontology, Dental School, University of Geneva, Geneva, Switzerland; ${ }^{6}$ Professor of Dental Material, ACTA, Amsterdam, The Netherlands *Correspondence to: Dr Stefano Ardu, Ecole de Médecine Dentaire, Université de Genève, Rue BarthélemyMenn 19, CH-1205 Genève, Switzerland Email:Stefano.Ardu@unige.ch

\section{Refereed Paper}

Accepted 28 November 2008

DOI: $10.1038 /$ sj.bdj.2009.116

${ }^{\circledR}$ British Dental Journal 2009; 206: 205-208
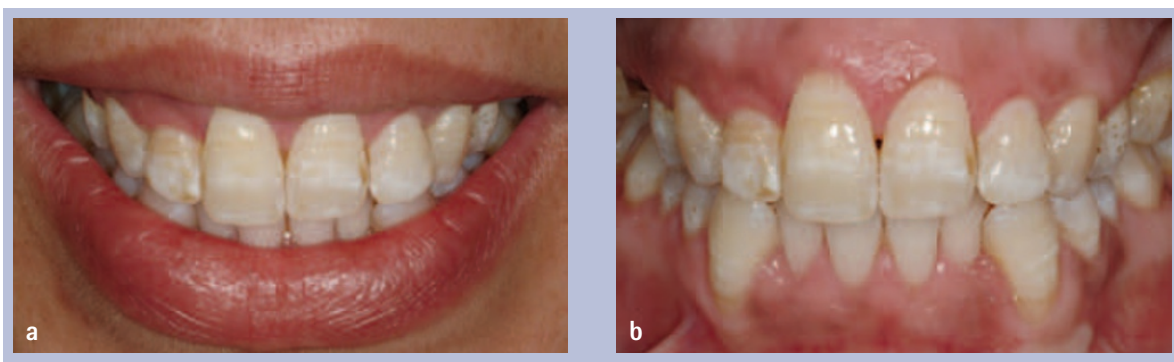

Fig. 1a-b Initial view of a patient affected by fluorosis



Fig. 1c Mega abrasion performed with a 60 um diamond bur

micro-abrasive treatments and resin composite restorations are widely used where, in the past, conventional prosthetic approaches were employed. Even if micro-abrasion is less expensive then a prosthetic approach, it can still be expensive due to its high chair time, especially in cases of medium to severe fluorosis where the treatment has to be repeated several times.

The aim of this article is to describe an easy technique for managing small superficial enamel defects such as

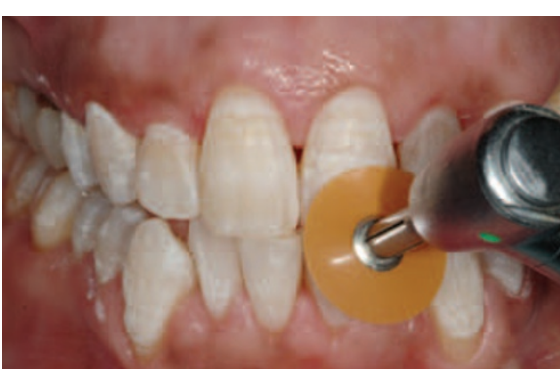

Fig. 1d Surface finishing with Sof-Lex ${ }^{\text {TM }}$ discs

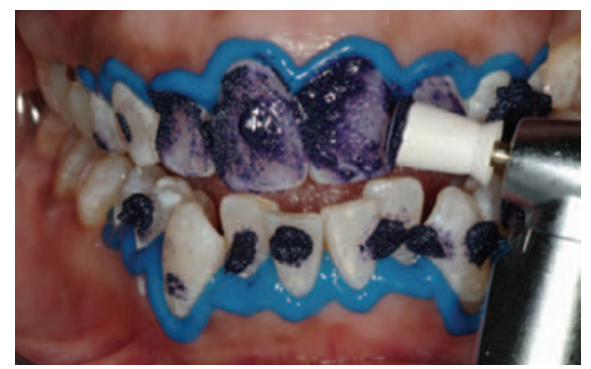

Fig. 1e Light curing rubber dam application and micro-abrasion with Opalustre ${ }^{T M}$

the ones present in medium to mediumsevere fluorosis. 


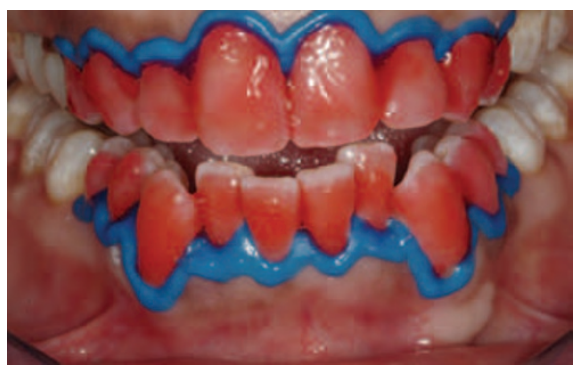

Fig. If Application of a power bleaching agent $\left(35 \% \mathrm{H}_{2} \mathrm{O}_{2}\right.$, Diva Smiles $\left.{ }^{\mathrm{T}}\right)$

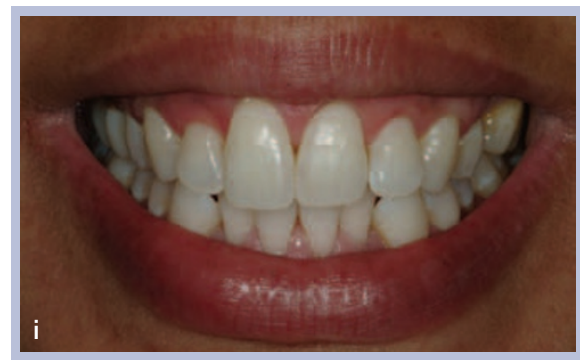

Fig. 1i-j Final view of the clinical case three months after the end of the treatment
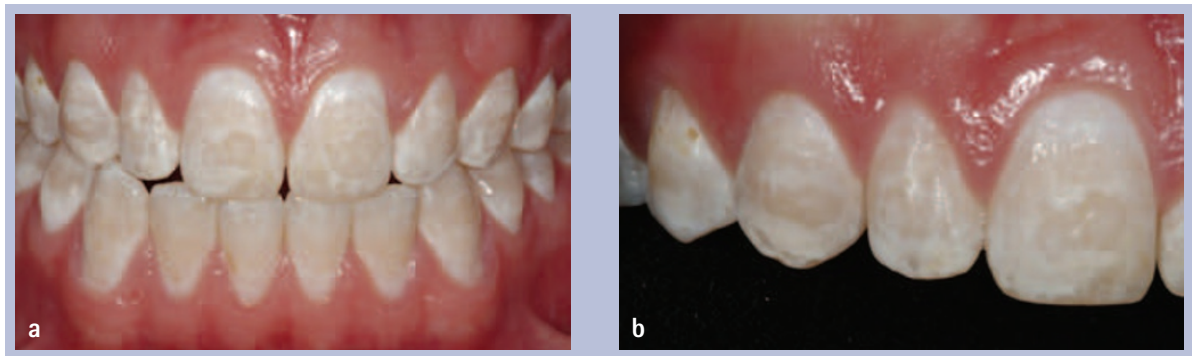



Fig. 1g Specific view of the bleaching agent action

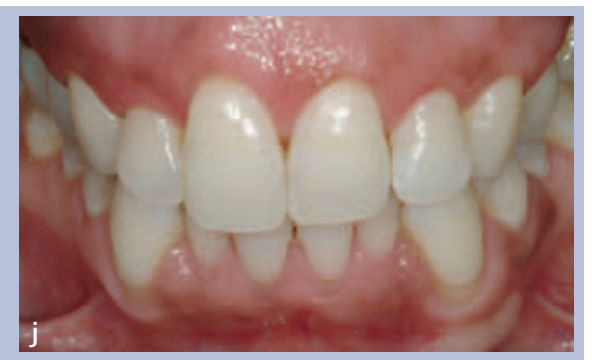

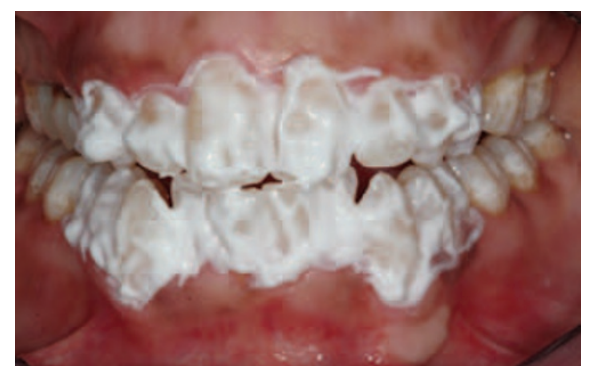

Fig. 1h Application of the desensitising agent (Diva Smiles ${ }^{\mathrm{TM}}$ )

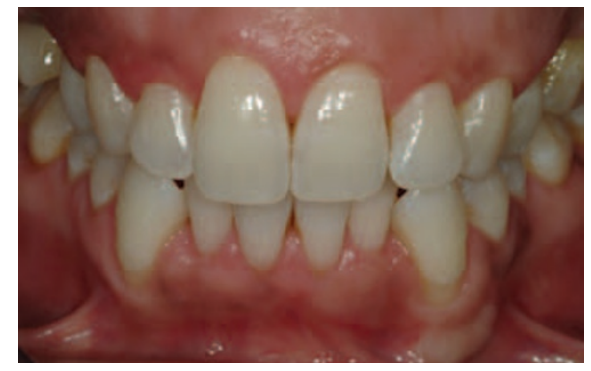

Fig. 1k Recall at 12 months

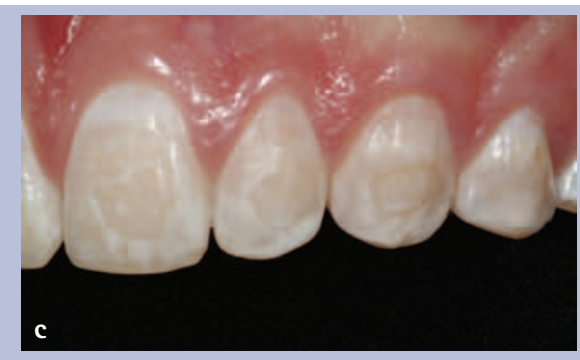

Fig. 2a-c Initial view of a patient affected by fluorosis and rests of luting composite after brackets debonding

\section{CLINICAL PROCEDURE}

The proposed technique is based on a combined chemico-mechanical approach which allows the management of enamel fluorosis affecting the more superficial part of the enamel. This approach is based on a combination of mega-abrasion, ${ }^{5}$ microabrasion $^{6}$ and power bleaching or home bleaching. ${ }^{7,8}$

Once a light to moderate EF (enamel fluorosis) is diagnosed (Figs 1a-1b, 2a-2c) by anamnesis and clinical examination, the patient is informed of this minimally invasive treatment option and the decision of finishing the treatment by in office bleaching or home bleaching is evaluated. Then a mechanical removal of the superficially affected enamel is carried out using a 60-80 $\mu \mathrm{m}$ diamond bur (D8GS bur Intensive SA CH-6916 Grancia, Switzerland) used with light pressure (Figs 1c and 2d). Once the superficial 200-400 enamel $\mu \mathrm{m}$ are removed, depending on the severity of the fluorosis ${ }^{4}$ a superficial enamel reshaping is performed aiming to recreate the macro-morphology and the micro-morphology of a sound tooth. For this aim medium to fine abrasive discs (Sof-lex 3M, St. Paul, USA, MN 551441000) or silicon points (Diatech Silicone Polishers Coltène/Whaledent AG 9450 Altstätten, Switzerland) can be used (Figs $1 \mathrm{~d}$ and 2e). Then after the application of a photo polymerisable rubber dam (Diva Smiles Kit, Marcs Group, Bahnhofstrasse 43 I 5000 Aarau, Switzerland) a microabrasion is performed. For this an abrasive paste containing silicon carbamide micro particles in water soluble paste and 6.6\% hydrochloric acid (Opalustre ${ }^{\circledR}$ Ultradent, South Jordan, Utah, USA) is employed. A layer of about $2-3 \mathrm{~mm}$ is applied on the affected teeth (Fig. 1e) and a specific rubber cup is used (Oralcups ${ }^{\mathrm{TM}}$ Opalustre ${ }^{\circledR}$ Ultradent, South Jordan, Utah, USA) attached to a gear reduction contra angle (Fig. 1e). The tooth surface is microabraded with slight pressure for about 60 to 120 seconds. Whenever necessary a small drop of water can be $\operatorname{added}^{9}$ and the abrasion can be repeated. In such a case, after each application an optical evaluation must be done after the water rinse, before proceeding to the next application. Then, to achieve more uniform tooth colour, ${ }^{10}$ if the patient has chosen a complete in office approach, power bleaching is performed (Figs 1f-1g) according to the manufacturer's instructions for 10-15 minutes (Diva Smiles Kit, Marcs Group, Bahnhofstrasse 43 I 5000 Aarau, Switzerland). After bleaching agent suction a second application can be performed if necessary followed by a thorough water rinsing and removal of the photo polymerisable rubber dam. Consequently the desensitising agent (Diva Smiles Kit, Marcs Group, Bahnhofstrasse 43 I 5000 Aarau, Switzerland) is applied (Fig. 1h), left undisturbed for ten minutes then suctioned.

When a home bleaching option is preferred, only a five minute topical fluoride application ${ }^{10}$ is performed and then the patient is dismissed after a two arch impression in order to provide bleaching trays and products at the following appointment. 


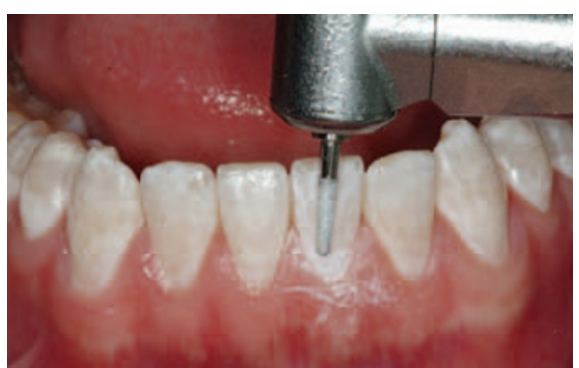

Fig. 2d Mega abrasion performed with a $60 \mu \mathrm{m}$ diamond bar

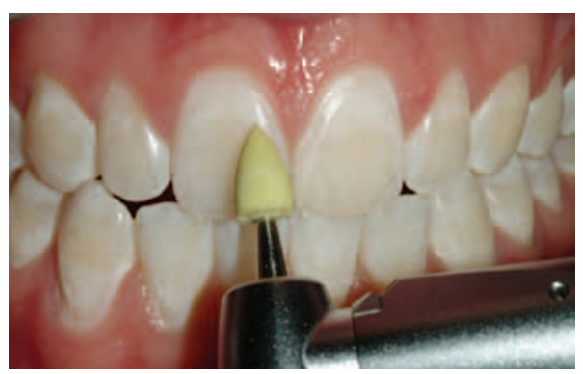

Fig. 2e Surface finishing with a silicon point
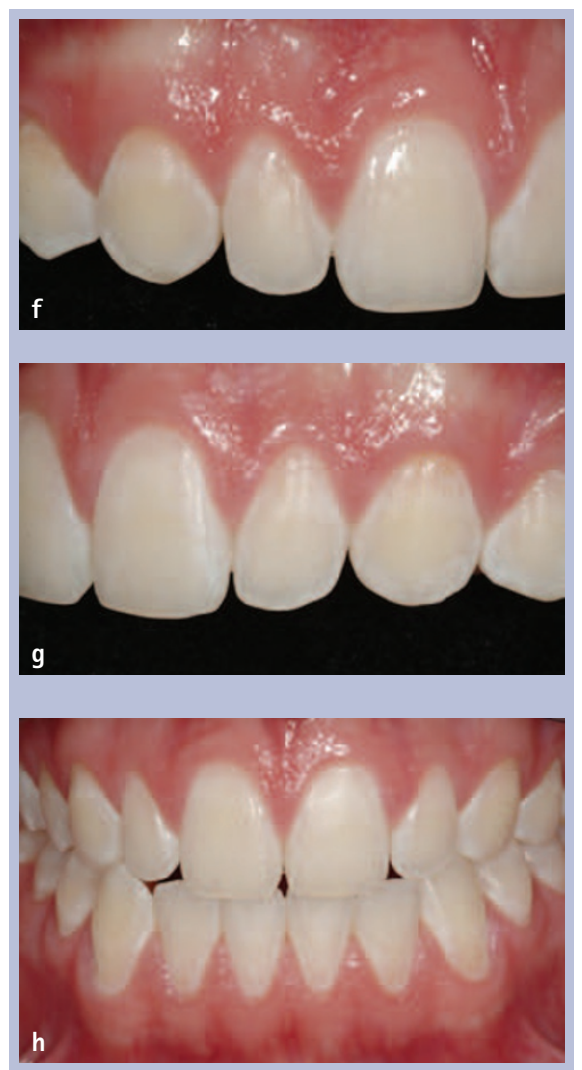

Fig. $2 f-h$ Final view three months after the end of the treatment

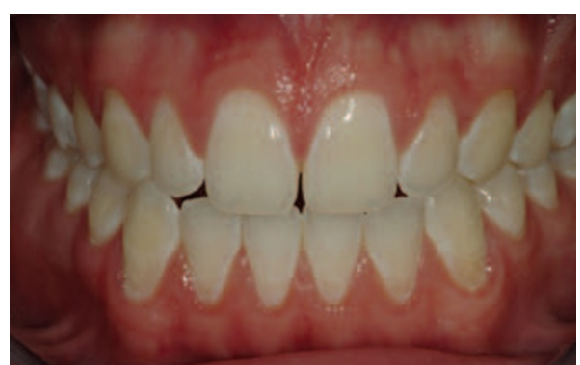

Fig. 2i Recall at 18 months
All aesthetic evaluations can be carried out only after a complete teeth rehydration (Figs 1i-1k, 2f-2i) and, if the result is not satisfactory, the complete treatment can be performed again.

\section{DISCUSSION}

Due to the recent increase in dental fluorosis ${ }^{2,3}$ extensive research has been performed to understand the aetiology and pathogenesis of this systemic disease. The aetiology currently seems to be well established ${ }^{1}$ and strictly based on the excessive consumption of fluoride during specific critical ages. This critical period, according to Browne, is 21-30 months of age for females and 1524 months of age for males. ${ }^{11}$ Regarding the mechanisms which are responsible for the development of the EF several theories have been proposed which can be summarised into a unique comprehensive concept.

The characteristic opacity of fluorotic enamel results from incomplete apatite crystal growth. Matrix proteins, which are associated with the mineral phase to permit a correct crystal growth, normally degrade and disappear during the enamel maturation phase. In fluorotic enamel, they are not eliminated resulting in their retention in the enamel tissue. Fluoride and magnesium concentrations increase while the carbonate level is reduced. Crystal surface morphology is slightly altered. Such changes in crystal chemistry and morphology, involving stronger ionic and hydrogen bonds, also lead to greater binding of modulating matrix proteins and proteolytic enzymes. This results in reduced degradation and enhanced retention of protein components in mature tissue. This is most likely responsible for porous fluorotic tissue, since complete matrix protein removal is necessary for a 'healthy' crystal growth. ${ }^{12}$ In other words fluorosed enamel is characterised by a retention of amelogenins in the early maturation stage of development and a consequent formation of a more porous enamel with a subsurface hypomineralisation. ${ }^{13}$ This can result in a whitish, brawny enamel aspect till the loss of enamel prisms, depending on EF severity.

In our technique we suggest starting with a mega-abrasion by a $60-80$ $\mu \mathrm{m}$ diamond bur in order to reduce the unaesthetic whitish enamel, minimising the clinical chair time. This approach is, in fact, less time-consuming then the more conventional micro-abrasion and, consequently, more advantageous for patients. Furthermore the unaesthetic loss of the micro and micro morphology typical of the deep micro-abrasive paste driven procedures ${ }^{9}$ can be avoided by simple enamel recontouring using the same diamond bur.

After completion of the micro-abrasion, a chairside (power) bleaching or a home bleaching technique can be indicated to hide residual whitish enamel and to better harmonise tooth colour.

Finally, the application of a desensitising paste based on fluoride and/or cppacp for 5 to 15 minutes is recommended. ${ }^{9}$ This approach is justified for two reasons. First it reduces the risk of post treatment sensitivity and second it protects teeth from possible external demineralisation. In Segura's experience, ${ }^{10}$ in fact, teeth treated with microabrasion followed by a four-minute application of 1\% neutral topical sodium fluoride exhibited significantly less enamel demineralisation when subjected to an artificial caries challenge than did teeth that underwent microabrasion alone, topical fluoride treatment alone, or no treatment at all.

\section{CONCLUSIONS}

This combined chemo-mechanical approach may be considered an interesting alternative to more invasive prosthetic techniques based on composite reconstructions or ceramic veneers and even chairside time if compared to the classical micro-abrasion. Furthermore, this new minimal invasive approach allows good aesthetic results and a possible cost reduction for patients.

We want to thank Marcs Group, Aarau, Switzerland for the kind supply of the bleaching material.

1. Browne $D$, Whelton $H, O^{\prime}$ Mullane D. Fluoride metabolism and fluorosis. J Dent 2005; 33: 177-186.

2. Whelton H P, Ketley C E, McSweeney F, O'Mullane $D M$. A review of fluorosis in the European Union: prevalence, risk factors and aesthetic issues. Com munity Dent Oral Epidemiol 2004; 32 (suppl 1).

3. Whelton H P, Clarke D, Daly F, McDermott S et al. Dental fluorosis in the Eastern Health Board in the republic of Ireland. Caries Res 1998; 32: 267-317.

4. Fejerskov 0, Manji F, Baelum V Moller I J. Dental fluorosis. A handbook for health workers. Copenhagen: Munksgaard, 1988. 
5. Magne P. Megabrasion: a conservative strategy for the anterior dentition. Pract Periodontics Aesthet Dent 1997: 9: 389-395.

6. Croll T P. Enamel microabrasion: the technique. Quintessence Int 1989; 20: 395-400.

7. Zaragoza V M T. Bleaching of vital teeth: technique. Estomodeo 1984; 9: 7-30.

8. Scherer W, Penugonda B, Styner D, Georgescu M. At-home vital bleaching: effects on stained enamel and dentin. Pract Periodontics Aesthet Dent 1992: 4: 11-15.

9. Ardu S, Stavridakis M, Krejci I. A minimally invasive treatment of severe dental fluorosis. Quintessence Int 2007; 38: 455-458.

10. Segura A, Donly K J, Wefel J S. The effects of microabrasion on demineralization inhibition of enamel surfaces. Quintessence Int 1997; 28: 463-466.
11. Browne $D$, Whelton $H, O$ 'Mullane D. Fluoride metabolism and fluorosis. J Dent 2005; 33: 177-186.

12. Robinson C, Connell S, Kirkham J, Brookes S J et al. The effect of fluoride on the developing tooth. Caries Res 2004; 38: 268-276

13. Buzalaf M A, Granjeiro J M, Damante C A, de Ornelas F. Fluoride content of infant formulas prepared with deionized, bottled mineral and fluoridated drinking water J Dent Child 2001: 68: 37-41. 